\title{
Heterosis and Combing Ability Studies for Yield and Yield Component Traits in Sunflower (Helianthus annuus)
}

\author{
Vikas V. Kulkarni ${ }^{1 *}$ and S.M. Supriya ${ }^{2}$ \\ ${ }^{1}$ AICRP on Sunflower, MARS, UAS - Raichur, Karnataka, India \\ ${ }^{2}$ Department of Genetics and Plant Breeding, AC, Dharwad, Karnataka, India \\ *Corresponding author
}

\section{A B S T R A C T}

\section{Keywords}

Sunflower,

Combining ability,

Heterosis, Gene

action.

\section{Article Info}

Accepted:

10 July 2017

Available Online:

10 September 2017
Major improvement in sunflower breeding requires heterotic hybrids which can be achieved by tapping combining ability of $\mathrm{F}_{1}$ hybrids developed from crossing of two genetically diverse female lines with male testers. In the present study, 56 hybrids were evaluated derived from four lines and fourteen testers' crosses in a line $\times$ tester mating design. The analysis of variance revealed existence of highly significant differences $(\mathrm{p}=0.05)$ among genotypes, parents and parents vs crosses indicating the presence of heterosis for quantitative traits in sunflower. The parental lines CMS-A6 and CMS-A2 and testers DOR-R3 and R-1F proved to be good combiners having the highest positive General Combing Ability (GCA) effect for yield (5.491**) and yield components traits and negative effect for days to $50 \%$ flowering $(-1.482 * *)$ and plant height $(-15.71 * *)$. The crosses CMS-A5 x R-103, CMS-A6 x R-103, CMS-A6 x R-7 and CMS-A1 x RGM-49 are promising hybrid combinations for seed yield as indicated by significant Specific Combing Ability (SCA) effects. These identified four hybrids can be taken to multi-location trials for further evaluation.

\section{Introduction}

Sunflower is highly cross pollinated crop and the main objective of sunflower breeding is to develop high yielding hybrid cultivars with stable and high yield through exploitation of heterosis. Sunflower hybrids are highly selffertile with better yielding capacity and more uniform in days to maturity (Seetharam, 1977; Kaya and Atakisi, 2004). Sunflower hybrids are genetically homogenous and uniform in plant height and growing period, resulting in decreased harvest losses. Another important advantage of hybrids over variety is easier insertion of genes for resistance to the most important sunflower diseases viz., Alternaria leaf spot, and powdery mildew.
Hybrid breeding programme in sunflower started with an aim to exploit the advantages of inter-allelic and intra-allelic interactions. However, crossing any two lines does not necessarily cause heterosis, since lines can be genetically related. Hence, it is prerequisite to estimate combining ability of all inbred lines before involving them in hybrid breeding programme.

In sunflower, heterosis breeding evolved successfully as the detection of cytoplasmic male sterility source (Leclercq, 1969) and fertility restoration (Kinman, 1970) that gave the required vigour to commercial hybrid seed 
production. In heterosis breeding programme, the selection of inbreds with good combining ability is very important for superior hybrid production. The estimation of general and specific combining ability helps to identify the potential parents in superior hybrid production for seed yield and oil contents. The line $\mathrm{x}$ tester analysis has been widely used for combining ability tests (Singh and Chaudhary, 1984, Patil et al., 2012) and an efficient method to assess the large number of inbreds as it provides the information on the relative importance of general combining ability effects and specific combining ability effects for yield and yield component traits.

To initiate hybrids breeding programme it is prerequisite to develop inbred lines with good combining ability. Information on GCA of lines to be used as parents as well as on their specific combining ability would be of immense value in formulating an efficient breeding strategy (Vikas et al., 2015). Combining ability studies elucidates the nature and magnitude of gene action involved in the inheritance of character by providing the information on the two components of variance viz., additive genetic variance and dominance variance, which are important to decide upon the parents and crosses to be selected for eventual success. Thus, this investigation was undertaken to determine magnitude of heterosis for yield and other quantitative traits and general and specific combining ability effects using $\mathrm{L} \times \mathrm{T}$ mating design.

\section{Materials and Methods}

The experiment was conducted at the experimental fields of Sunflower Scheme at Main Agricultural Research Station, UAS, Raichur during 2014-15. The experimental material used in the present study consisted of four lines (CMS-A1, CMS-A2, CMS-A5 and CMS-A6) and fourteen testers (RGM-39,
RGM-41, RGM-49, RGM-69, RGM-27, EC602060, GMU-520, R-103, DOR-R3, R-1-F, R-7, R-393, R-127 and R-8). The objective of this investigation was to examine, the general and specific combining abilities of newly developed sunflower inbred lines using line $\mathrm{x}$ tester analysis (Kempthorne, 1957) and to estimate the amount of heterosis in fifty six hybrids. The crossing block consisting of 4 lines and 14 testers was raised during kharif2014. Crossing was undertaken in a line $\mathrm{x}$ tester fashion using 4 lines and 14 testers at Main Agricultural Research Station, Raichur. A total of 56 hybrids were raised along with 18 parents and three checks viz., RSFH-130, KBSH-44 and GK-202 during Rabi-2014-15 in a randomized block design with two replications. Observations were recorded in each entries on randomly selected five plants for seven characters viz., plant height $(\mathrm{cm})$, days to 50 per cent flowering, head diameter $(\mathrm{cm})$, test weight (g/100 seeds), volume weight $(\mathrm{g} / 100 \mathrm{ml})$ and oil content (\%).However, the seed yield/ plant (g) was calculated by using plot yield data.

\section{Results and Discussion}

\section{Analysis of variance}

The production of sunflower can greatly be increased through the cultivation of promising hybrids. The evolution of desirable hybrids can only be possible by crossing potential female lines with male testers. Presence of genetic variability is the basic requirement for developing high yielding and better adapted hybrids in sunflower.

The mean sums of square due to lines were found to be highly significant only for plant height a considerable amount of variation existing for this trait among female lines which can be exploitation for combining ability analysis. The mean sum of squares for testers was not significant for all the seven 
characters studied except for plant height. However, line $\mathrm{x}$ tester interaction was highly significant for all characters studied suggesting contribution of SCA effects towards variation among the crosses.

\section{General Combining Ability (GCA) and Specific Combining Ability (SCA) effects}

Line $\times$ tester analysis can evaluate more number of genotypes as compared to diallel and partial diallel mating designs. This technique can be used even when the inbred lines have self-incompatibility and male sterility where diallel crosses entirely fail to use such abnormal conditions. The success of any breeding programme largely depends on selection of suitable parental lines. Information regarding different types of gene action, relative magnitude of genetic variance and combining ability estimates are important genetic parameters for the improvement of sunflower (Sher et al., 2009). The presence of non-additive genetic variances and effects, presumably dominant genes are the primary justification for initiating the hybrid development programme in sunflower. Higher GCA effects are more desirable for selfpollinated crops and varieties released as pure lines while, SCA is more important for the production of hybrids in cross pollinated crops (Hallauer and Miranda, 1986).

\section{General combining ability effects}

Minimum number of days to flowering is preferred to reduce crop growth period to accommodate cropping system. The sunflower growers require short duration hybrids coupled with high seed yields, because such hybrids reduce the incidence of insect-pest, disease attack and adverse environmental effects. Main focus in sunflower breeding is therefore diverted to develop early maturing hybrids or openpollinated cultivars for short growing cycle
(Sher et al., 2009). The general combing ability analysis revealed that line CMS-A2 (0.429 ) is the best general combiners for earliness which recorded significantly negative GCA effects for days to $50 \%$ flowering, while CMS-A6 (-9.156) exhibited desirable negative GCA effects for plant height. Among fourteen testers evaluated, six (RGM-39, RGM-69, GMU-520, DOR-R3, R$1-F)$ and seven (RGM-69, GMU-520, DORR3, R-1-F, R-7, R-393 and R-127) testers exhibited negative desirable GCA effects for days to $50 \%$ flowering and plant height, respectively (Table 2) indicating the importance of additive gene action advocating both these characters. These results indicated that earlier and shorter hybrids could be developed by crossing good general females with male lines having highly negative GCA effects. Significantly negative GCA effects for days to $50 \%$ flowering were also reported by Patil et al., 2017, Kang et al., (2013) and Saleem et al., (2014) while Ahmad et al., (2011) observed significantly negative GCA for plant height; hence these identified parents can be considered as good general combiners for developing dwarf/semi-tall and early hybrids in sunflower.

Achieving higher commercial yield is the basic objective for most of the breeding programmes in any of the crop plants, where more number of photosynthetically active leaves play major role. Higher number of leaves plant $^{-1}$ would lead to more photosynthetic activity, ultimately higher seed yields, while bigger heads inflicts direct effect on number of seeds head ${ }^{-1}$, consequently imposes significant positive effect on seed yield (Zia-Ullah et al., 2013). The GCA estimates revealed that female lines CMS$\mathrm{A} 1(0.735)$ and CMS-A2 (0.910) and testers RGM-49 (1.206), GMU-520 (0.856) and R-1F (0.831) manifested significantly higher positive GCA effects for head diameter; whereas two lines (CMS-A6, CMS-A1) and 
seven testers (R-127, R-393, RGM-49, R-103, RGM-69, DOR-R3 and RGM-39) for seed yield plant ${ }^{-1}$; and two lines (CMS-A6 and CMS-A5) and four testers (DOR-R3, R-127 and EC-602060) for test weight. Considerably high positive GCA effects with predominantly additive genes suggested that such females lines can be crossed with good general combiner testers to develop potential hybrids, synthetics and composite varieties to improve head diameter (Machikowa et al., 2011); seeds yield (Hladni et al., 2011); test weight and volume weight (Andarkhor et al., 2012). Mirarab and Ahmadikhah (2010) stated that parents which were good general combiners for economic traits might be extensively used in hybridization programmes so as to identify suitable parents for hybridization and developing potential hybrids for 1,000-achene weight and seed yield.

The female lines and testers with higher positive GCA estimates are desirable parents to be used for developing sunflower hybrids with improved oil content (Kang et al., 2013; Saleem et al., 2014). Most of the characters studies were controlled by additive gene action as indicated by higher GCA effects in sunflower.

\section{Specific combining ability effects}

The hybrids which expressed significantly negative SCA effects for days to maturity and plant height contribute favourable additive genes for earliness and shorter plant height (Patil et al., 2017). Out of eleven $F_{1}$ hybrids exhibiting higher negative SCA effects for day's maturity and plant height, at least three hybrids CMS-A1 x DOR-R3, CMS-A2 x R127 and CMS-A1 x GMU-520 were identified consistent in expressing significantly higher desirable negative SCA effects for both the traits. Results indicated that these crosses possess dominant or over dominant type of genes with decreasing effect hence may be exploited for reduced number of days to flowering and plant height (Table 3). Fifteen out of 56 hybrids, exhibited significantly positive SCA effects for head diameter, 10 for seed plant $^{-1}$; 12 for test weight; 9 hybrids for volume weight; 18 hybrids for seed yield per plant and 4 for oil content (Table 3). The hybrids which manifested significantly positive SCA effects for yield were not consistently higher for all the traits, yet the hybrids viz., CMS-A5 x R-103 (head diameter, test weight, volume, seed yield per plant), CMS-A1 x RGM-39(seed yield, head diameter, volume weight and test weight) CMS-A2 $\times$ RGM-39(head diameter, yield and oil content) and CMS-A6 x R-8 (for seed yield and test weight) expressed relatively higher positive SCA effects indicating that these characters are influenced by dominant and over dominant type of genes.

In consonance to our findings, Saleem et al., 2014 and Karasu et al., 2010 reported higher positive SCA for seed yield and yield related traits, Abdullah et al., 2010 for head diameter; Andarkhor et al., 2013 for test weight and volume weight; and Kang et al., 2013 for oil content. The heterotic performance of hybrid combinations depends upon the combining abilities of their parents (Kadkol et al., 1984). Kaya and Atakisi (2004) reported that superior hybrids were obtained by crossing females and males with high GCA and SCA effects.

The lines CMS-A1 and CMS-A2 (head diameter), CMS-A5 and CMS-A6 (test weight) and CMS-A1 and CMS-A6 (seed yield) were found to be good general combiner for improving yield. The data revealed that, the female parents CMS-A6 was best general combiner for four characters (plant height, seed yield, oil content and test weight) followed by CMS-A2 for three characters (days to $50 \%$ flowering and head diameter). 
Table.1 ANOVA for seven characters in 56 hybrids of sunflower

\begin{tabular}{|c|c|c|c|c|c|c|c|c|}
\hline Character & Df & $\begin{array}{l}\text { Plant height } \\
\text { (cm) }\end{array}$ & $\begin{array}{l}\text { Days to } \\
\mathbf{5 0 \%} \\
\text { flowering }\end{array}$ & $\begin{array}{l}\text { Head } \\
\text { diameter } \\
(\mathrm{cm})\end{array}$ & $\begin{array}{l}\text { Test } \\
\text { weight } \\
\text { (g) }\end{array}$ & $\begin{array}{l}\text { Volume } \\
\text { weight } \\
\text { (g/100ml) }\end{array}$ & $\begin{array}{l}\text { Seed Yield } \\
\text { (g/plant) }\end{array}$ & $\begin{array}{l}\text { Oil } \\
\text { content } \\
(\%)\end{array}$ \\
\hline Replication & 1 & 0.056 & 0.036 & 1.181 & 0.189 & 0.039 & 4.400 & $42.324 * * *$ \\
\hline Crosses & 55 & $624.624 * * *$ & $9.958 * * *$ & $12.805 * * *$ & $1.102 * * *$ & $8.284 * * *$ & $136.617 * * *$ & $7.759 * * *$ \\
\hline Lines & 3 & $1045.532 * * *$ & 2.452 & 29.474 & 2.745 & 12.533 & 86.152 & 11.853 \\
\hline Testers & 13 & $970.527 *$ & 5.690 & 4.440 & 1.066 & 6.993 & 151.737 & 8.923 \\
\hline Line V/s Tester & 39 & $476.946 * * *$ & $11.959 * * *$ & $14.312 * * *$ & $0.987 * * *$ & $8.387 * * *$ & $135.458 * * *$ & $7.056 * * *$ \\
\hline
\end{tabular}

$*, * *$-indicates significance at $5 \%$ and $1 \%$ level respectively

Table.2 General combining ability effects of the parents on different characters

\begin{tabular}{|c|c|c|c|c|c|c|c|c|}
\hline $\begin{array}{l}\text { Sl. } \\
\text { No. }\end{array}$ & Parents & $\begin{array}{l}\text { Plant height } \\
\text { (cm) }\end{array}$ & $\begin{array}{l}\text { Days to } \\
50 \% \\
\text { flowering }\end{array}$ & $\begin{array}{l}\text { Head } \\
\text { diameter } \\
(\mathrm{cm})\end{array}$ & $\begin{array}{l}\text { Test weight } \\
\text { (g) }\end{array}$ & $\begin{array}{l}\begin{array}{l}\text { Volume } \\
\text { weight } \\
(\mathrm{g} / 100 \mathrm{ml})\end{array} \\
\end{array}$ & $\begin{array}{l}\text { Seed } \\
\text { Yield } \\
\text { (g/plant) }\end{array}$ & $\begin{array}{l}\text { Oil content } \\
(\%)\end{array}$ \\
\hline \multicolumn{9}{|c|}{ Lines } \\
\hline L1. & CMS-A1 & $3.451 * *$ & $0.250 * *$ & $0.735 * *$ & $-0.439 * *$ & $-0.531 *$ & $0.575^{*}$ & $-0.932 * *$ \\
\hline L2. & CMS-A2 & $2.862 * *$ & $-0.429 * *$ & $0.910 * *$ & 0.000 & -0.385 & -0.332 & 0.038 \\
\hline L3. & CMS-A5 & $2.844 * *$ & 0.071 & $-1.290 * *$ & $0.171 * *$ & -0.038 & $-2.214 * *$ & 0.470 \\
\hline L4. & CMS-A6 & $-9.156^{* *}$ & 0.107 & -0.354 & $0.268 * *$ & $0.954 * *$ & $1.971 * *$ & 0.424 \\
\hline T1. & RGM-39 & $16.790 * *$ & $-0.982 * *$ & $-1.056^{* *}$ & $-0.691 * *$ & -0.656 & $-3.146^{* *}$ & 0.124 \\
\hline T2. & RGM-41 & $3.165 * *$ & $1.643 * *$ & -0.444 & $-0.691 * *$ & -0.144 & $-5.346 * *$ & $-2.001 * *$ \\
\hline T3 & RGM-49 & $14.290 * *$ & $1.268 * *$ & $1.206 * *$ & $-0.241 *$ & -0.794 & $3.916 * *$ & $0.974 *$ \\
\hline $\mathrm{T} 4$ & RGM-69 & $-2.335 * *$ & $-0.357^{*}$ & -0.069 & -0.079 & -0.881 & $2.316 * *$ & $0.899 *$ \\
\hline T5 & RGM-27 & $15.165 * *$ & 0.268 & 0.281 & -0.066 & $0.819 *$ & 0.804 & 0.586 \\
\hline T6 & EC_602060 & $6.040 * *$ & 0.268 & 0.606 & $0.371 * *$ & $1.244 * *$ & 0.191 & 0.186 \\
\hline $\mathrm{T} 7$ & GMU-520 & $-15.710 * *$ & $-0.607 * *$ & $0.856^{*}$ & 0.059 & $-1.319 * *$ & $1.316^{*}$ & 0.199 \\
\hline $\mathrm{T} 8$ & R-103 & 1.478 & 0.268 & 0.344 & 0.184 & 0.594 & $3.554 * *$ & $-1.101 *$ \\
\hline T9 & DOR-R3 & $-13.960 * *$ & $-0.482 * *$ & $-1.219 * *$ & $0.471 * *$ & $-1.244 * *$ & $2.166 * *$ & $-1.214 * *$ \\
\hline T10 & $\mathrm{R}-1 \mathrm{~F}$ & $-7.335 * *$ & $-1.482 * *$ & $0.831^{*}$ & -0.129 & $1.544 * *$ & $5.091 * *$ & -0.151 \\
\hline T11 & $\mathrm{R}-7$ & $-7.835 * *$ & 0.268 & $-0.856^{*}$ & -0.066 & -0.669 & $5.491 * *$ & $-0.958 *$ \\
\hline T12 & R-393 & $-14.335 * *$ & 0.268 & -0.469 & 0.146 & 0.656 & $-9.071 * *$ & $1.636^{* *}$ \\
\hline T13 & R-127 & $-1.835^{*}$ & -0.732 & 0.081 & $0.396 * *$ & 0.544 & $-2.459 * *$ & -0.501 \\
\hline T14 & R-8 & $6.415^{* *}$ & $0.393 *$ & -0.094 & $0.334 * *$ & 0.306 & $-4.821 * *$ & $1.324 * *$ \\
\hline
\end{tabular}


Table.3 Specific combining ability effects of the hybrids on different characters

\begin{tabular}{|c|c|c|c|c|c|c|c|c|}
\hline $\begin{array}{l}\text { Sl. } \\
\text { No. }\end{array}$ & Parents & $\begin{array}{c}\text { Plant } \\
\text { height } \\
(\mathrm{cm})\end{array}$ & $\begin{array}{c}\text { Days to } \\
\mathbf{5 0 \%} \\
\text { flowering }\end{array}$ & $\begin{array}{c}\text { Head } \\
\text { diameter } \\
(\mathrm{cm})\end{array}$ & $\begin{array}{c}\text { Test } \\
\text { weight }(g)\end{array}$ & $\begin{array}{c}\text { Volume } \\
\text { weight } \\
\text { (g/100ml) }\end{array}$ & $\begin{array}{c}\text { Seed } \\
\text { Yield } \\
\text { (g/plant) } \\
\end{array}$ & $\begin{array}{c}\text { Oil } \\
\text { content } \\
(\%)\end{array}$ \\
\hline & Lines & & & & & & & \\
\hline 1. & CMSA1xRGM-39 & $14.674 * *$ & $1.375 * *$ & $2.028 * *$ & $0.427 *$ & $3.456 * *$ & $8.250 * *$ & $-2.193 *$ \\
\hline 2. & CMSA1xRGM-41 & $4.799 * *$ & $-1.750 * *$ & -0.685 & -0.123 & 0.794 & $14.450 * *$ & -0.668 \\
\hline 3. & CMSA1xRGM-49 & $6.174 * *$ & $1.625 * *$ & -0.835 & $0.477^{*}$ & $2.094 *$ & $6.238 * *$ & 0.257 \\
\hline 4. & CMSA1xRGM-69 & $26.799 * *$ & $1.250 * *$ & 0.040 & $-0.536 * *$ & -0.469 & $-3.263 * *$ & 0.632 \\
\hline 5. & CMSA1xRGM-27 & $-19.201 * *$ & $.1 .375^{* *}$ & 0.490 & 0.052 & $-3.019 * *$ & $-5.400 * *$ & -0.956 \\
\hline 6. & CMSA1xEC_602060 & $-7.576 * *$ & $-2.375^{* *}$ & 0.265 & -0.086 & -0.694 & $-2.337 *$ & -0.006 \\
\hline 7. & CMSA1x GMU-520 & $-14.826 * *$ & $-2.500 * *$ & $-1.885^{*}$ & $2.177 * *$ & -0.781 & 0.087 & 1.432 \\
\hline 8. & CMSA1x R-103 & $22.987 * *$ & $2.625 * *$ & 0.328 & $-1.048 * *$ & $-2.544 * *$ & $-12.000 * *$ & -1.168 \\
\hline 9. & CMSA1x DOR-R3 & -32.576 & $-1.625 * *$ & $-4.410 * *$ & $0.564 * *$ & 1.244 & $5.238 * *$ & 0.694 \\
\hline 10 & CMSA1xR-1F & $-3.201 *$ & $-0.625^{*}$ & $-2.060 * *$ & 0.064 & 0.006 & -1.288 & -0.118 \\
\hline 11 & CMSAx R-7 & $-6.701 * *$ & $-2.375 * *$ & $1.828^{*}$ & 0.152 & $1.969 *$ & -4.188 & $5.213 * *$ \\
\hline 12 & CMSA1x R-393 & $-3.701 *$ & $2.625 * *$ & 0.740 & $-1.361 * *$ & 0.094 & $-6.525 * *$ & 0.044 \\
\hline 13 & CMSA1xR-127 & $19.799 * *$ & $0.625^{*}$ & $3.290 * *$ & $-0.661 * *$ & -1.244 & 0.813 & $-1.918^{*}$ \\
\hline 14 & CMSA1xR-8 & $-7.451 * *$ & $2.500 * *$ & 0.865 & -0.098 & -0.906 & -0.075 & -1.243 \\
\hline 15 & CMSA2x RGM-39 & $-11.237 * *$ & $1.554 * *$ & $4.593 * *$ & -0.113 & $-1.690 *$ & $4.157 * *$ & $3.513 * *$ \\
\hline 16 & CMSA2x RGM-41 & $-17.112 * *$ & $3.929 * *$ & $-4.160 * *$ & -0.162 & -0.253 & $-3.443 * *$ & -1.238 \\
\hline 17 & CMSA2x RGM-49 & -0.737 & $-0.696^{*}$ & 0.090 & $-0.562 * *$ & -0.753 & -0.855 & 1.037 \\
\hline 18. & CMSA2x RGM-69 & -3.112 & -0.071 & 0.065 & -0.325 & $-1.815^{*}$ & $-2.155^{*}$ & -0.138 \\
\hline 19 & CMSA2x RGM-27 & -14.112 & $-1.696 * *$ & -0.785 & 0.363 & -0.765 & $4.957 * *$ & 1.125 \\
\hline 20 & CMSA2xEC_602060 & $16.013 * *$ & $4.304 * *$ & $2.390 * *$ & 0.025 & 0.460 & $6.170 * *$ & -1.025 \\
\hline 21 & CMSA2xGMU-520 & $-6.737 * *$ & $-1.821 * *$ & -0.460 & $-0.463 *$ & -1.378 & -0.855 & -0.238 \\
\hline 22 & CMSA2x R-103 & $-6.674 * *$ & $-1.696 * *$ & $-5.047 * *$ & $-0.637 *$ & $-1.690 *$ & $-17.643 * *$ & -0.488 \\
\hline 23 & CMSA2x DOR-R3 & $25.013 * *$ & $1.054 * *$ & $2.065 * *$ & $0.425 *$ & 0.947 & $2.195^{*}$ & 1.025 \\
\hline 24 & CMSA2x R-1F & $19.888 * *$ & $1.554 * *$ & $1.865^{*}$ & 0.325 & 0.410 & -0.430 & -0.338 \\
\hline 25 & CMSA2x R-7 & $-11.112 * *$ & $-1.696^{* *}$ & $-1.547 *$ & 0.263 & 1.522 & -0.430 & 0.168 \\
\hline
\end{tabular}




\begin{tabular}{|c|c|c|c|c|c|c|c|c|}
\hline 26 & CMSA2xR-393 & 0.888 & $-0.696^{*}$ & $1.665^{*}$ & 0.050 & $2.297 * *$ & -0.318 & 0.775 \\
\hline 27 & CMSA2x R-127 & $-15.112 * *$ & $-4.696 * *$ & $-3.985 * *$ & $0.500 * *$ & 1.160 & -0.830 & $2.212 *$ \\
\hline 28 & CMSA2x R-8 & $24.138 * *$ & $0.679 *$ & $2.890 * *$ & 0.312 & 1.547 & $9.482 * *$ & 0.637 \\
\hline 29 & CMSA5x RGM-39 & 2.281 & $-4.696 * *$ & $-5.497 * *$ & $-0.384^{*}$ & $-3.637 * *$ & $-5.861 * *$ & $4.955^{* *}$ \\
\hline 30 & CMSA5x RGM-41 & $15.406 * *$ & $0.679 *$ & $2.940 * *$ & 0.166 & -0.599 & -1.111 & 1.380 \\
\hline 31 & CMSA5x RGM-49 & 1.281 & $-4.446^{* *}$ & 1.090 & $0.416^{*}$ & 0.301 & 0.727 & -0.445 \\
\hline 32 & CMSA5x RGM-69 & $-17.594 * *$ & -0.571 & -0.635 & $0.554 * *$ & 0.938 & $9.927 * *$ & -0.920 \\
\hline 33 & CMSA5x RGM-27 & $22.406 * *$ & 0.304 & 0.115 & 0.091 & $3.288 * *$ & $-6.111 * *$ & -1.408 \\
\hline 34 & CMSA5xEC_602060 & -1.969 & $-1.071 * *$ & -0.710 & 0.004 & 1.563 & $-2.498 *$ & 0.292 \\
\hline 35 & CMSA5x GMU-520 & $10.781 * *$ & $4.304 * *$ & 0.240 & $-1.034 * *$ & 0.926 & -1.023 & $-1.870^{*}$ \\
\hline 36 & CMSA5xR-103 & $-13.406 * *$ & $0.696^{*}$ & $3.353 * *$ & $0.891 * *$ & $1.913^{*}$ & $19.189 * *$ & 1.480 \\
\hline 37 & CMSA5x DOR-R3 & $-3.969 *$ & $3.679 * *$ & 1.015 & $-0.846^{* *}$ & $2.251 * *$ & $-11.423^{* *}$ & 1.092 \\
\hline 38 & CMSA5x R-1F & $-17.094 * *$ & -0.196 & -1.235 & 0.204 & $-1.837 *$ & $5.702 * *$ & 0.530 \\
\hline 39 & CMSA5x R-7 & $12.406 * *$ & $1.054 * *$ & $2.453 * *$ & $-0.409 *$ & $-2.974 * *$ & $-2.548 *$ & $-2.664 * *$ \\
\hline 40 & CMSA5x R-393 & -2.594 & -0.446 & $-3.035 * *$ & $1.179 * *$ & 0.599 & $5.564 * *$ & -0.658 \\
\hline 41 & CMSA5x R-127 & $-4.094 *$ & $2.304 * *$ & -0.685 & 0.079 & -1.487 & $-11.198 * *$ & -0.370 \\
\hline 42 & CMSA5x R-8 & $-3.844^{*}$ & $-1.196^{* *}$ & 0.590 & $-0.909 * *$ & -0.049 & 0.664 & -1.395 \\
\hline 43 & CMSA6x RGM-39 & $-5.719 * *$ & $0.696^{*}$ & $-1.483 *$ & 0.070 & $1.871 *$ & $-6.546 * *$ & 0.751 \\
\hline 44 & CMSA6x RGM-41 & -3.094 & $-2.321 * *$ & $1.904^{* *}$ & 0.120 & 0.058 & $-9.896^{* *}$ & 0.526 \\
\hline 45 & CMSA6x RGM-49 & $-6.719 * *$ & $1.518 * *$ & -0.346 & -0.330 & $-1.642 *$ & $-6.109 * *$ & -0.849 \\
\hline 46 & CMSA6x RGM-69 & $-6.094 * *$ & $-1.607 * *$ & 0.529 & 0.307 & 1.346 & $-4.509 * *$ & 0.426 \\
\hline 47 & CMSA6x RGM-27 & $10.906 * *$ & $-1.232 * *$ & 0.179 & $-0.505 * *$ & 0.496 & $6.554 * *$ & 1.239 \\
\hline 48 & CMSA6xEC_602060 & $-6.469 * *$ & $-1.232 * *$ & $-1.946 * *$ & 0.057 & -1.329 & -1.334 & 0.739 \\
\hline 49 & CMSA6x GMU-520 & $10.781 * *$ & $.643^{*}$ & $2.104 * *$ & $-0.680 * *$ & 1.233 & 1.791 & 0.676 \\
\hline 50 & CMSA6xR-103 & -2.906 & $-0.732 *$ & 1.367 & $0.795 * *$ & $2.321 * *$ & $10.454 * *$ & 0.176 \\
\hline 51 & CMSA6x DOR-R3 & $11.531 * *$ & -0.482 & 1.329 & -0.143 & $-4.442 * *$ & $3.991 * *$ & $-2.811 * *$ \\
\hline 52 & CMSA6xR-1F & 0.406 & -0.482 & $1.429 *$ & $-0.593 * *$ & 1.421 & $-3.984 * *$ & -0.074 \\
\hline 53 & CMSA6xR-7 & $5.406 * *$ & $1.768 * *$ & $-2.733 * *$ & -0.005 & -0.517 & $7.166^{* *}$ & $-2.717 * *$ \\
\hline 54 & CMSA6xR-393 & $5.406 * *$ & $-0.732 *$ & 0.629 & 0.132 & $-1.792 *$ & 1.279 & -0.161 \\
\hline 55 & CMSA6xR-127 & -0.594 & $4.768 * *$ & 1.379 & 0.082 & 1.571 & $11.216^{* *}$ & 0.076 \\
\hline 56 & CMSA6xR-8 & -12.844 & $-0.857 * *$ & $-4.346^{* *}$ & $0.695 * *$ & -0.592 & $-10.071^{* *}$ & $2.001 *$ \\
\hline
\end{tabular}


Table.4 Crosses exhibiting maximum sca effects, their mean performance and gca status of parents with respect seven characters in sunflower

\begin{tabular}{|c|c|c|c|c|c|}
\hline \multirow{2}{*}{ Characters } & \multirow{2}{*}{ Desirable crosses } & \multirow{2}{*}{ SCA } & \multirow{2}{*}{ Mean } & \multicolumn{2}{|c|}{ GCA study of } \\
\hline & & & & Female & Male \\
\hline \multirow{3}{*}{ Plant height $(\mathrm{cm})$} & CMSA1xRGM-27 & -19.201 & 127.500 & Low & Low \\
\hline & CMSA5x RGM-69 & -17.594 & 111.000 & Low & High \\
\hline & CMSA2x RGM-41 & -17.112 & 117.000 & Low & Low \\
\hline \multirow{3}{*}{$\begin{array}{l}\text { Days to } 50 \% \\
\text { flowering }\end{array}$} & CMSA2x R-127 & -4.696 & 52.000 & Low & High \\
\hline & CMSA5x RGM-39 & -4.696 & 52.500 & Low & High \\
\hline & CMSA5x RGM-49 & -4.446 & 59.500 & Low & High \\
\hline \multirow{3}{*}{$\begin{array}{l}\text { Head diameter } \\
\qquad(\mathrm{cm})\end{array}$} & CMSA2x RGM-39 & 4.593 & 26.200 & High & Low \\
\hline & CMSA5xR-103 & 3.353 & 23.800 & High & Low \\
\hline & CMSA1xR-127 & 3.290 & 25.500 & High & Low \\
\hline \multirow{2}{*}{ Test weight (g) } & CMSA1x GMU-520 & 2.177 & 6.800 & Low & Low \\
\hline & CMSA5x R-393 & 1.179 & 6.500 & High & Low \\
\hline \multirow{3}{*}{$\begin{array}{l}\text { Volume weight } \\
\text { (g/100ml) }\end{array}$} & CMSA1xRGM-39 & 3.456 & 38.450 & Low & High \\
\hline & CMSA5x RGM-27 & 3.288 & 40.250 & Low & Low \\
\hline & CMSA6xR-103 & 2.321 & 40.050 & Low & High \\
\hline \multirow{3}{*}{$\begin{array}{l}\text { Seed yield/Plant } \\
(\mathrm{g})\end{array}$} & CMSA5xR-103 & 19.189 & 55.050 & Low & High \\
\hline & CMSA1xRGM-41 & 14.450 & 44.200 & High & Low \\
\hline & CMSA6xR-103 & 11.216 & 45.250 & High & Low \\
\hline \multirow{3}{*}{ Oil content $(\%)$} & CMSAx R-7 & 5.213 & 36.175 & Low & Low \\
\hline & CMSA5x RGM-39 & 4.955 & 38.400 & Low & Low \\
\hline & CMSA2x RGM-39 & 3.513 & 29.500 & Low & Low \\
\hline
\end{tabular}

GCA: General Combining Ability SCA: Specific Combining Abilit

Among the testers, DOR-R3 and R-1-F was best general combiner for four characters (plant height, head diameter, test weight, yield and plant height, volume weight and yield, respectively) followed by RGM-69 andR-7 for three characters (plant height, seed yield, oil content and plant height, head diameter and seed yield, respectively). Based on total positive and significant GCA scores, it is observed that CMS-A1 among lines and RGM-49 among testers were the good general combiners. The above results are in agreement with Reddy and Madhavi Latha (2005).
For most of the characters studied, the low $\mathrm{x}$ low GCA combination of parents were noticed in better performing heterotic cross combinations (Table 4), indicating the predominance of non-additive gene action as reported by Kadkol et al., (1984); Reddy and Madhavi Latha (2005). The significant positive SCA effects were noticed in 16 hybrids for seed yield. Among them CMS-A5 $x$ R-103(19.189) which was followed by CMS-A1 x RGM-41 (14.450), CMS-A6 x R127 (11.216) and CMS-A6 x R-103 (10.454) recorded highest positive SCA effects. 
Contribution of lines, testers and lines $\times$ testers interaction to total variance

The female lines had contributed normal effect for plant height and seeds yield, hence exhibited fair amount of maternal influence (Table 1). While the tester parents played very important role towards plant height and seed yield manifested significant paternal effect with additive genes for these traits. Line $\times$ tester interactions contributed relatively more as compared to female and male lines, however high values were noted for plant height, followed seeds yield and head diameter, which showed preponderance of dominant genes for these traits.

\section{Heterosis}

Heterosis is a universal phenomenon which occurs both in self and cross pollinated crop species like sunflower. Heterosis is the superiority of $F_{1}$ over the mean of the parents or over the better parent or over the standard commercial check (Hayes et al., 1956). The maximum utilization of heterosis is possible when the variance due to both additive and non additive gene actions are fully exploited since they play a significant role in determining the magnitude of expression of yield and its component characters. In India, the era of sunflower hybrids began with the production and release of first ever sunflower hybrid BSH-1 by Seetharam, (1981).

Several workers as Habib (2009) and Vikas et al., (2015) reported the importance of plant height in contributing higher seed yield as it is significantly associated with seed yield in sunflower. In present study, 33 hybrid combinations recoded significant positive heterosis ranging from 6.16 to $43.56 \%$ and only seven hybrids exhibited negative heterosis. The maximum heterosis percentage for plant height was displayed by the crosses CMS-A2 x R-8, CMS-A5 x RGM-27 and CMS-A2 x EC-602060 with a value of 43.56,
41.00 and 36.00 , respectively in positive direction. Whereas, the hybrids CMS-A1 $\mathrm{x}$ DOR-R3 (-34.87) and CMS-A1 x GMU-520(22.61) recorded highly significant heterosis in negative direction. Positive average heterosis combined with negative standard heterosis indicates that experimental hybrids are taller than their parents. The positive and significant heterobeltiosis was manifested by 11 cross combinations for plant height. The hybrids viz., CMS-A1 x RGM-39, CMS-A2 x R-8 and CMS-A1 x R-103 showed the highest positive heterobeltiosis with the values of 18.12, 16.61 and $11.83 \%$, respectively. However, CMS-A1 $x$ DOR-R3, CMS-A1 x GMU-520 and CMSA5 x R-1-F exhibited negatively significant heterobeltosis. Many workers have reported both positive and negative heterosis values for plant height. The predominance of tallness over dwarfness indicates tallness to be dominant character as reported by Patil et al., (2017), Vikas et al., (2015) and Habib et al., (2009) reported negative heterosis. However, Vulpe (1967) did not observe heterosis for plant height.

The variation for days to 50 per cent flowering ranged from 52 days (CMS-A2 $\mathrm{x}$ R-127) to 63 days (CMS-A5 x EC-602060) in hybrids. The hybrids CMS-A5 x RGM-39, CMS-A5 x R-1-F, CMS-A5 x R-8, CMS-A5 x R-127 and CMS-A5 x RGM-69 recorded highly significant heterosis in negative direction. Larger head size is a desired trait to affect more yield in sunflower crop. In the present study out of 56 crosses, all the crosses exhibited positive heterosis. The maximum heterosis percentage $(148.34,146.99$ and $145.90 \%$ ) for head diameter was displayed by the crosses CMS-A2 x RGM-39, CMS-A5 $\mathrm{x}$ RGM-41 and CMS-A6 x RGM-41, respectively. The positive and significant heterobeltiosis was manifest 52 cross combinations for head diameter. Hybrids viz. CMS-A2 x RGM-39, CMS-A2 x EC-602060 and CMS-A2 $x$ R-8 showed the highest positive heterobeltiosis with the values of 
$112.15,104.86$ and $103.24 \%$, respectively for head diameter. Patil et al., (2017) and Patil et al., (2012) also found high positive heterosis for head diameter.

Heterosis studies for 100-seed weight revealed that 13 cross combinations showed positive heterosis over the better parent value. The cross combinations CMS-A6 x RGM-41, CMS-A5 x RGM-41 and CMS-A1 x GMU520 displayed the highest heterosis percentages (100.00, 97.87 and $78.95 \%)$ for 100-test weight. Whereas, positive and significant heterobeltiosis $(58.54,52.44$ and $48.68 \%$ ) was observed in the crosses, CMSA5 x R-393, CMS-A5 x R-103 and CMS-A2 $\mathrm{x}$ R-8, respectively. High mid parent heterosis was also recorded by Gangappa et al., (1997). The results are in line with the findings of Kandhola et al., (1995) regarding heterobeltiosis. A total of nine hybrids recorded significant positive heterosis over better parent for volume weight. Among them the hybrids viz., CMS-A5 $\mathrm{x}$ RGM-27 followed by CMS-A1 x RGM-39 and CMSA2 x R-393, recorded positive highly significant heterosis over the better parents. These observations were in line with the reports of, Virupakshappa (1991) and Sugoor (1992), Patil et al., (2012) and Patil et al., (2017).

Among the 56 hybrid, 49 crosses exhibited highly significant positive heterosis for seed yield. The maximum heterosis percentage for seed yield per plant was manifested by the crosses CMS-A5 x R-103, CMS-A6 x R-103, CMS-A6 x R-7 and CMS-A1 x RGM-49, with a value of $163.40 \%, 141.63 \%, 126.50 \%$ and $120.73 \%$, respectively. The positive and highly significant heterobeltiosis was manifested by 40 cross combinations. The hybrids viz., CMS-A5 x R-103, CMS-A6 x R103, CMS-A6 x R-7 and CMS-A5 x RGM-69 showed the highest positive heterobeltiosis with the values of $119.32 \%, 92.38 \%, 87.24 \%$ and $77.49 \%$, respectively for seed yield. The presence of non-additive gene action in determining seed yield per plant has also been reported by several workers. It augurs well for the development of potential high yielding hybrids. Similar conclusions have been drawn by Goksoy et al., (2000), Patil et al., (2012) and Patil et al., (2017). Reports of negative heterosis for seed yield are also on record viz., Naresh et al., (1996), Kumar et al., (1999) and Yadav et al., 2009..

Highest oil content heterosis was indicated in CMS-A5 x RGM-39, CMS-A1 x R-7, CMSA6 x R-8, CMS-A1 x R-393 and CMS-A1 x GMU-520 hybrids with a value of 22.10, $17.45,13.49,9.09$ and 8.93 respectively. Prominent heterobeltiosis was observed in CMS-A5 x RGM-39, CMS-A6 x R-8 and CMS-A1 x R-7 for oil content in descending order. The results get support from the earlier findings of Kandhola et al., (1995), Rather and Sandha (1999) and Nehru (2000).

Positive heterosis for oil yield was displayed in 35 crosses. Ten cross combinations showed significant heterosis for oil yield. Maximum heterosis was recorded in CMS-A5 x RGM39, CMS-A1 x R-7, CMS-A6 x R-8 with a value of $22.10,17.45$ and 9.09 , respectively for oil yield. Positive heterobeltiosis for oil yield was recorded in 24 crosses. Positive and significant increase over better parent was observed in ten hybrids for sunflower oil yield. Maximum heterobeltiosis for oil yield was recorded in CMS-A5 x RGM-39 followed by CMS-A6 x R-8 with the value of 19.81 and $8.77 \%$, respectively. Rather and Sandha (1999) observed a wide range heterosis and heterobeltiosis in their respective studies. It is, therefore, concluded hybrid CMS-A5 x RGM-39 can be exploited for better oil yield on commercial basis.

\section{References}

Abdullah, K., Mehmet, OZ., Mehmet, S., Abdurrahim, G. and Metin, Z., 2010, 
Combining ability and heterosis for yield and yield components in sunflower. Not Botanicae Hort Agrobot Cluj-Napoca. 38: 259-264.

Ahmad, W., Ahmed, S. and Tahir, N., 2011, Combining ability analysis for achene's yield and related traits in sunflower (Helainthus annuus L.). Chil J. Agri.c Res., 72: 21-26.

Andarkhor, S. A., Mastibege, N. and Rameeh, V., 2012, combining ability of agronomic traits in sunflower (Helianthus annuus L.) using line $\times$ tester analysis. Int. J. Biol., $4: 89-95$

Andarkhor, S.A., Rameeh, V. and Alitabar, R. A., 2013, Estimation of genetic parameters for yield components and seed yield in sunflower using line $x$ tester analysis. Afr. J. Biotechnol., 12:3978-3983

Gangappa, E., Channakrishniah, K. M., Ramesh, S. and Harini, A. S., 1997, Exploitation of heterosis in sunflower (Helianthus annuus L.). Crop Res., 13: 339-348.

Goksoy, A. T., Turkec, A. and Turan, Z. M., 2000, Heterosis and combining ability in sunflower (Helianthus annuus L.). Ind. J. Agric. Sci., 70(8): 525-529.

Hallauer, A. R., and Miranda, J. B., 1986, Quantitative genetics in maize breeding. Iowa State University Press, Ames, pp 267-294.

Hayes, H. K., Immer, F. F. and Smith, D. L., 1956, Methods of Plant Breeding. McGraw Hill Book Publishing Company, Inc., New Delhi, pp. 21-34.

Hladni, N. D., Skoric, M.K., Balalic, S., Jocic, V., Miklic, A. and Dusanic, N., 2011, Lines $\times$ tester analysis for yield components in sunflower and their correlations with seed yield (Helianthus annииs L.). Genetica, 43:297-306.

Kadkol, G.P., Anand, I. J. and Sharma, R. P., 1984, Combining ability and heterosis in sunflower. Indian J. Genet. Pl.
Breed, 44(3): 447-451.

Kandhola, S. S., Behl, R. K. and Punia, M. S., 1995, combining ability in sunflower. Ann. Biol., 11: 103-106.

Kang, S. A., Khan, F. A., Ahsan, M. Z., Chatha, W. S. and Saeed, F., 2013, Estimation of combining ability for the development of hybrid genotypes in sunflower (Helianthus annuus L.). $J$. Biol. Agric. Healthc., 3: 68-74.

Karasu, A., Mehmet, O. Z., Sincik, M., Goksoy, A. T. and Turn, Z. M., 2010, Combining ability and heterosis for yield and yield components in sunflower. Not Bot Hort Agrobot Cluj. 38:259-264.

Kaya, Y., and Atakisi, I., 2004, combining ability analysis of some yield characters of sunflower (Helianthus annuus L.). Helia, 47:75-84.

Kempthorne, O., 1957, Introduction to genetic statistics. John Wiley and Sons Inc, New York.

Kumar, A. A., Ganesh, M., Kumar, S. S. and Reddy, A. V., 1999, Heterosis in sunflower (Helianthus annuus L.). Ann. Agric. Res., 20(4): 478-480.

Machikowa, T., Chiraporn, S. and Kiattisak, F., 2011, General and specific combining ability for quantitative characters in sunflower. J. Agric. Sci., 3: 91-95.

Mirarab, M., and Ahmadikhah, A., 2010, Study on genetics of some important phonological traits in rice using line $x$ tester. Ann. Biol. Res., 1:119-125.

Naresh, R., Channakrishnaiah, K. M. and Gangappa, E., 1996, Heterosis in single cross and three way cross hybrids of sunflower. Mysore J. Agric. Sci., 30: 197-203.

Nehru, S.D., A Manjunath and Basavarajaiah, D., 2000, Extent of heterosis for seed yield and oil content in sunflower. Karnataka J. Agri. Sci., 13(3): 718-720. 
Patil Rudragouda, Vikas V. Kulkarni, Mallikarjun Kenganal, Shankergoud, I. and Diwan, J. R., 2017, Combining ability studies in restorer lines of sunflower (Helianthus annuus L.). Journal of Applied and Natural Science 9 (1): 603 - 608

Patil, R., Shaker Goud, I., Vikas Kulkarni and Banakar C., 2012, combining ability and gene action studies for seed yield and its components in Sunflower (Helianthus annuus L.). Electronic Journal of Plant Breeding, 3(3): 861867

Rather, A. G., and Sandha, G. S., 1999, Heterosis in sunflower. Advances in Pl. Sci., 12(1): 53-56.

Reddy, A., and Madhavilatha, K., 2005, combining ability for yield and yield components in sunflower. J. Res., Acharya N. G. Ranga Agric. Univ., 33(2): 12-17.

Saleem, U. D., Khan, M. A., Gull, S., Usman, K., Saleem, F. Y. and Siyal, O. U., 2014, Line $\times$ tester analysis of yield and yield related attributes in different sunflower genotypes. Pak. J. Bot., 46: 659-665.

Seetharam, A., 1981, Hybrid sunflower for higher yields. Seeds and Farms, 6: 2729.

Seetharam, A., Kusumakumari, P., Patil, N. M. and Singagi, S. S., 1977, Performance of hybrids of sunflower produced by means of cytoplasmic male sterility. SABRAO J., 9: 51-55.

Sher, A. K., Habib, A., Ayub, K., Muhammad, S., Shah, M, K. and
Bashir, A., 2009, Using line $\times$ tester analysis for earliness and plant height traits in sunflower (Helianthus annuus L.). Recent Res. Sci. Technol., 1: 202206.

Sugoor, R. K., 1992, Heterosis and combining ability of induced mutant restorer lines in sunflower (Helianthus annuus L.). M. Sc. (Agri.) Thesis, Univ. Agric. Sci., Dharwad, Karnataka, India.

Vikas Kulkarni, Shankegoud, I. and Govindappa, M.R., 2015, Evaluation and characterization of sunflower germplasm accessions for quantitative characters. Electronic Journal of Plant Breeding, 6(1): 257-263.

Virupakshappa, K., 1991, Heterosis, combining ability and stability analysis in sunflower (Helianthus annuus L.). $M$. Sc. (Agri.) Thesis, Univ. Agric. Sci. Dharwad, India.

Vulpe, V., 1967, Study of some sunflower hybrids produced through male sterility and fertility restoration. Analel Institutului Cerceyari Pentu Ceeale Plantae Fundulea, 34: 391-399.

Yadav, H. K., Sudhir, S. and Singh. S. P., 2009, Genetic combining ability estimates in the $\mathrm{F} 1$ and $\mathrm{F}_{2}$ generations for yield, its component traits and alkaloid content in opium poppy (Papaver somniferum L.). Euphytica, 168:23-32.

Zia-Ullah, Z., Sadaqat, H. A., Tahir, M. H.N and Sadia, B., 2013, Path coefficient analysis of various traits in sunflower (Helianthus annuus L.). J. Glob Innov. Agric. Soc. Sci., 1: 5-8.

\section{How to cite this article:}

Vikas V. Kulkarni and Supriya, S.M. 2017. Heterosis and Combing Ability Studies for Yield and Yield Component Traits in Sunflower (Helianthus annuus). Int.J.Curr.Microbiol.App.Sci. 6(9): 3346-3357. doi: https://doi.org/10.20546/ijcmas.2017.609.413 